\title{
HACIA UNA TIPOLOGÍA DEL DiÁlOgo JUDICIAL TRANSNACIONAL
}

TOWARDS A TYPOLOGY OF TRANSNATIONAL JUDICIAL DIALOGUE

\section{RODRIGO BRITO MELGAREJO ${ }^{1}$}

RESUMEN: Este artículo ofrece una tipología sobre la forma en que los jueces constitucionales usan precedentes extranjeros al resolver casos, a fin de determinar si al recurrir a resoluciones de tribunales de otros países los órganos jurisdiccionales actúan de manera apropiada en su labor de interpretación constitucional, así como para determinar cuándo recurren a esta herramienta interpretativa y cuáles son las implicaciones y parámetros en cada caso.

PALABRAS CLAVE: diálogo judicial, precedentes extranjeros, jueces constitucionales, interpretación constitucional.

ABSTRACT: This article provides a typology of the uses of foreign precedents by constitutional judges in deciding cases in order to provide insight into whether these precedents can be appropriately used in constitutional interpretation, when and what the stakes and parameters are in each case.

KeyworDs: judicial dialogue, foreign precedents, constitutional judges, constitutional interpretation.

Sumario: I. Comunicación judicial y proceso dialógico. II. Los factores que favorecen el diálogo entre los tribunales. III. La importancia del diálogo entre tribunales. IV. Elementos para una tipología del diálogo judicial transnacional. V. Conclusiones. VI. Fuentes.

1 Licenciado y Doctor en Derecho por la Universidad Nacional Autónoma de México, así como Doctor en Justicia Constitucional y Derechos Fundamentales por la Universidad de Pisa. Profesor en la Facultad de Derecho de la UNAM. <r.brito@icloud.com>. ORCID: <https://orcid.org/0000-0003-0786-1543>.

Fecha de recepción: 10 de diciembre de 2018; fecha de aprobación: 9 de febrero 2019. 


\section{Comunicación Judicial y proceso dialógico}

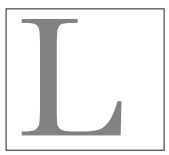

a forma en que entendemos la protección de los derechos ha cambiado radicalmente a partir de la apertura que los jueces constitucionales han tenido respecto de otras experiencias en los últimos años. Cada vez son más los órganos de control que voltean la mirada hacia la forma en que otros tribunales están resolviendo casos similares a los que ellos deben enfrentarse, generándose así una comunicación transjudicial a partir de la generación de una especie de diálogo que encuentra uno de sus fundamentos más sólidos en la idea de que la emergencia de problemas que en su evolución, pero, sobre todo, en su solución posible tienen una dimensión y una naturaleza que supera los confines estatales, muchas veces impone a los actores que participan en este nuevo escenario buscar respuestas en criterios que no sólo se encuentran en las normas nacionales. Surge entonces la necesidad de un intercambio de ideas entre las distintas jurisdicciones nacionales que tiende a provocar una convergencia en los distintos sistemas jurídicos. Este hecho ha sido producto de una serie de fenómenos que favorecen la circulación de principios comunes (al menos en los ordenamientos liberal-democráticos) y, al mismo tiempo, dotan a estos principios de una fuerza expansiva que les permite convertirse en referentes aplicables, bajo ciertas condiciones, en otros sistemas.

Esa situación ha provocado una dicotomía caracterizada por planteamientos que parecieran contraponerse, pero que, dado el contexto actual, conviven dotando de cierta complejidad a los sistemas jurídicos. Por un lado, existen elementos ideológicos, religiosos, históricos, económicos, climáticos, geográficos, sociológicos y culturales que determinan una identidad nacional sobre la que se construyen normas e instituciones que regulan la vida de los individuos; pero por otra parte, los seres humanos, debido a las concurrencias que se presentan en el mundo jurídico, van acogiendo cada vez 
con mayor fuerza una concepción universal de la idea de justicia y, en consecuencia, consideran difícil justificar la existencia de respuestas diversas a problemas idénticos. ${ }^{1}$ La dicotomía planteada ha provocado un aumento en el interés por la forma en que son tratados los casos que se resuelven en otros países, lo que tiene como consecuencia que, en la práctica jurídica interna de los Estados, las resoluciones dictadas por los órganos de control de otros países sean revalorizadas.

La llamada "globalización judicial", por tanto, se afianza día con día a través de distintos canales de comunicación que los jueces constitucionales utilizan para encontrar soluciones mejor argumentadas en los procesos que se ven obligados a resolver. ${ }^{2}$ De esta forma, a través de una mayor comunicación con otros órganos jurisdiccionales, cada vez son más los tribunales que interactúan entre sí, no sólo tomando como referencia los casos resueltos por otros jueces o en otras instancias, sino también ofreciendo sus propios razonamientos para participar en un proceso que está cambiando de la simple recepción al diálogo entre órganos jurisdiccionales. ${ }^{3}$ Este proceso dialógico ha adquirido mayor fuerza debido a que los problemas comunes y las necesidades globales que enfrentan los Estados, han hecho crecer la necesidad de conocer no sólo las reglas escritas o la doctrina que se crea en el mundo académico, sino también las reglas operacionales, los modelos de sentencia y las técnicas

1 Canivet, Guy, "La pratica del diritto comparato nelle corti supreme", en Markesinis, Basil y Fedtke, Jörg, coords., Giudici e diritto straniero. La pratica del diritto comparato, Bolonia, Il Mulino, 2009, pp. 214 ss.

2 La globalización judicial, según Slaughter, describe un proceso de interacción judicial que supera fronteras al intercambiar ideas y cooperar en casos que implican tanto el derecho nacional como el internacional. Véase Slaughter, Anne-Marie, "Judicial globalization", Virginia fournal of International Law, vol. 40, 1999-2000, p. 1104.

${ }^{3}$ L'Heureux-Dubé, Claire, "The importance of dialogue: Globalization and the international impact of the Rehnquist Court", Tulsa Law Fournal, vol. 34, 1998, pp. 17 ss. 
de razonamiento de los que se valen otros órganos jurisdiccionales. El diálogo, entonces, busca acabar con cualquier autismo por parte de unos tribunales que en nuestros días deben necesariamente interpretar los derechos en red por la misma naturaleza transversal y global de su objeto. ${ }^{4}$ De hecho, son muchos los factores que, cada vez con mayor fuerza, fomentan la práctica dialógica entre los órganos jurisdiccionales.

II. Los FACTORES QUE FAVORECEN EL DIÁLOGO ENTRE LOS TRIBUNALES

Hoy en día son muchos los factores que hacen posible el diálogo entre los tribunales constitucionales. Esos factores pueden ser de carácter general o propios del país en el que se intenta poner en práctica la comunicación entre órganos jurisdiccionales y, en no pocas ocasiones, influyen considerablemente en la práctica judicial. Entre los factores generales, la migración de las ideas constitucionales y la expansión del control de constitucionalidad pueden considerarse como elementos que favorecen el diálogo transjudicial. El primero de estos elementos es fundamental, pues cada vez son más los países que comparten valores liberal-democráticos y, en consecuencia, gran parte de las instituciones y concepciones que éstos generan. Ciertas ideas cardinales como la protección de los derechos humanos, la división de poderes, el pluralismo, etc., son tomadas y compartidas por muchos Estados, lo que ha dado como resultado la creación de una concepción axiológica de los textos constitucionales en la que se marcan contenidos mínimos para que una Constitución pueda ser considerada como democrática. ${ }^{5}$

4 García Roca, Javier, "El diálogo entre el Tribunal Europeo de Derechos Humanos y los tribunales constitucionales en la construcción de un orden público europeo", Teoría y Realidad Constitucional, núm. 30, 2012, pp. 192-195.

5 En este sentido Ferrajoli, Luigi, Principia Iuris. Teoria del diritto e della democrazia, t. 1, Laterza, Roma-Bari, 2007, pp. 859 ss. 
Ahora bien, por lo que respecta al segundo elemento es necesario señalar que al extenderse la idea de que hablar de un Estado liberal-democrático significa también referirse a ciertos estándares mínimos de carácter constitucional y a la existencia de un sistema de control con el que se asegure la vigencia de la norma fundamental, el número de tribunales constitucionales que pueden considerarse verdaderamente democráticos aumentó de manera importante en las últimas décadas. Además, si se toma en cuenta que muchos de estos órganos de control han comenzado a trabajar bajo la nueva perspectiva que aportó el constitucionalismo de la posguerra y que su jurisprudencia (producto en algunos casos de varias décadas de experiencia) se ha basado en estas ideas, es evidente que el escepticismo hacia el material que ofrece la comparación jurídica comienza a diluirse. ${ }^{6}$

Una consecuencia de la circulación de tópicos en materia constitucional y del surgimiento de un buen número de tribunales constitucionales, es que éstos deben enfrentar problemas muy similares, lo que favorece el diálogo entre diversos órganos de control. En el ámbito de los derechos humanos, por ejemplo, diversos órganos jurisdiccionales se han pronunciado sobre temas como el aborto, la libertad de expresión, las prácticas religiosas y la libertad de conciencia o la equidad de género. Además, las jurisdicciones constitucionales se han ocupado también de cuestiones estructurales como el federalismo y la distribución del poder. Estas materias, son sólo una muestra de la manera en que, al revisar temas constitucionales, los órganos jurisdiccionales de control están tratando problemas parecidos que pueden servir como referentes en la labor interpretativa que llevan a cabo. ${ }^{7}$

6 Cfr. Ackerman, Bruce, "The rise of world constitutionalism", Yale Law School Occasional Papers, núm. 4, 1996, p. 2.

7 Véase en este sentido Barak, Aharon, "A judge on judging. The role of a Supreme Court in Democracy", en Harvard Law Review, núm. 116, 2002-2003, p. 110 . 
Las semejanzas en estos temas tienen al menos dos fuentes: ${ }^{8}$ un compromiso con las normas constitucionales comunes y la necesidad de aplicarlas a desarrollos culturales, sociales, políticos y económicos comparables. Esto porque aun cuando muchos de los sistemas constitucionales que existen reflejan importantes diferencias relacionadas con la lengua, con su estructura o su historia, generalmente están comprometidos con los mismos principios básicos del Estado constitucional. En el ámbito de los derechos fundamentales esta situación se acentúa debido a que existen textos que pueden considerarse modélicos (como la Declaración de los Derechos del Hombre y del Ciudadano de 1789 o la Declaración de Derechos norteamericana de 1797) y que han influenciado un gran número de constituciones o han sido parte fundamental en la elaboración de declaraciones de derechos e instrumentos de carácter internacional que son vinculantes en muchos países y que han servido también como fuentes de inspiración en la concepción de instrumentos regionales de protección de derechos. ${ }^{9}$ En pocas palabras, muchas veces los ciudadanos de distintos Estados encuentran garantizados similares derechos, que las instituciones deben proteger actuando de manera similar (aunque con los matices que emergen de las diferencias propias de cada nación).

Debe considerarse también que, en ocasiones, los Estados no sólo comparten los mismos principios básicos dentro de sus sistemas constitucionales, sino que también afrontan los mismos retos al aplicar esos principios a la realidad de la cultura contemporánea, pues en este siglo el desarrollo económico y tecnológico, los cambios demográficos, los asuntos políticos, sociales, culturales y religiosos, así como los sucesos mundiales, a menudo rebasan las fronteras na-

8 Cfr. Rahdert, Mark C., "Comparative constitutional advocacy", en American University Law Review, vol. 56, núm. 3, 2006, pp. 565.

9 Rosenfeld, Michael, "Constitutional migration and the bounds of comparative analysis", New York University Annual Survey of American Law, vol. 67, 2001, pp. 69 ss. 
cionales creando la misma clase de fricciones constitucionales. Por este motivo, los desarrollos jurisprudenciales frecuentemente toman senderos similares.

Por otra parte, los jueces constitucionales utilizan con frecuencia los mismos procesos analíticos al tratar los casos a los que se enfrentan. Conceptos como ponderación, proporcionalidad o racionalidad, se presentan como instrumentos interpretativos que se usan en diversos países. Es verdad que en ocasiones estos principios tienen significados o aplicaciones diversas en los distintos sistemas de control que existen o que muchas veces pueden encontrarse otros que no se ven reflejados en figuras afines en otros países; sin embargo, es un hecho que hoy en día puede hablarse de la existencia de una base común en los procesos de análisis dentro de los sistemas de control.

Si a esto sumamos que los tribunales constitucionales están alcanzando niveles de profesionalismo cada vez más homogéneos, el uso del método comparativo como herramienta en la interpretación se hace más simple. Además, los intercambios y encuentros entre jueces de distintas naciones, así como los congresos y simposios sobre el tema organizados en el mundo académico, favorecen también el diálogo entre los tribunales constitucionales.

Debe decirse además, que otros factores generales que contribuyen al uso del método comparativo en la interpretación son la creación de organizaciones instituciones internacionales que ofrecen y analizan distintos materiales de derecho comparado, el tratamiento que se da a este tema en las escuelas de derecho, así como la creciente accesibilidad a las decisiones de los tribunales constitucionales y la creación de oficinas, dentro de la organización de estas instituciones jurisdiccionales, que dan seguimiento a las decisiones de otros órganos de control.

Ahora bien, por lo que se refiere a los factores propios de cada Estado que favorecen el diálogo judicial transnacional, es necesario comentar que las naciones con sistemas de control incipiente, por 
ejemplo, pueden ser más propensas al diálogo debido a que el uso del derecho comparado como herramienta o método interpretativo puede dotar de legitimidad a las decisiones de sus tribunales constitucionales y constituir una fuente importante de referencias tanto en lo que concierne a los procesos decisionales como a sus consecuencias. Esto, sin embargo, no implica que en el diálogo participen sólo aquellos países que buscan tomar prestados conceptos o argumentos que han sido construidos por los tribunales de las democracias consolidadas, pues en muchas ocasiones también toman parte en él aquellos Estados que cuentan con una tradición jurídica fuerte y un sistema jurídico lo suficientemente afianzado como para resolver los casos que se les presentan, pero que, a pesar de ello, intentan saber en qué forma han sido resueltos casos similares fuera de sus fronteras.

Es evidente entonces que la apertura al diálogo dependerá de muchos factores de carácter interno (v. gr. Preparación de los jueces constitucionales, apertura normativa al derecho comparado, procedimientos de control, factores históricos, etc.) y que determinarán su propensión a la deliberación comparativa. Lo que es importante subrayar es que en la mayor o menor apertura de los órganos jurisdiccionales de control al uso del derecho comparado no solamente influyen factores externos de carácter general, sino también aspectos particulares que determinarán la factibilidad y los costos que eventualmente puede implicar para los jueces constitucionales el uso del método comparativo como canon de interpretación.

Ahora bien, si es cierto que cada vez son más los factores que favorecen el diálogo transjudicial, no puede pasarse por alto que la idea de diálogo jurisdiccional es una noción que, aunque no es nueva, sí puede considerarse bastante imprecisa y, para convertirse en una verdadera categoría jurídica en vez de una simple intuición, es necesario que se ofrezcan elementos que permitan a los operadores jurídicos comprender de mejor manera los alcances y bondades de las construcciones argumentativas basadas en procesos 
dialógicos. Por ello, en las páginas siguientes se ofrecerán algunos insumos para la construcción de una tipología de comunicación judicial, atendiendo a la manera en que puede emplearse el diálogo judicial transnacional.

\section{LA IMPORTANCIA DEL DIÁLOGO ENTRE TRIBUNALES}

La palabra diálogo encuentra entre las acepciones contempladas en el Diccionario de la Real Academia Española, la imagen de "discusión o trato en busca de avenencia". ${ }^{\circ}$ De esto se deduce de manera bastante evidente, como señala Laurence Burgorgue-Larsen, que el diálogo induce tanto el acuerdo como la oposición, tanto la contradicción o la discordia como el acuerdo, la concordia o la aprobación. ${ }^{11}$ Ahora bien, para los órganos jurisdiccionales, el concepto de diálogo conlleva, ante todo, la necesidad de justificar sus resoluciones fundamentándolas en argumentos suficientes. Esto es así pues:

Tradicionalmente, los tribunales decidían principalmente a partir de su autoridad, por lo que argumentaban poco sus resoluciones. No se veían llamados a sustentar la solución adoptada, ni tampoco justificaban que fuera la mejor entre otras opciones jurídicamente posibles. En cambio, en el nuevo derecho público, los tribunales deben argumentar para convencer a otros actores jurídicos y justificar la toma de determinada decisión. Tal justificación representa un elemento primordial en este modelo de derecho público, pues la

10 Estos señalamientos corresponden a la tercera acepción de la palabra, consultable en <www.rae.es>.

11 Burgorgue-Larsen, Laurence, "La formación de un derecho constitucional europeo a través del diálogo judicial", en Alejandro Saiz Arnaiz y Eduardo Ferrer Mac-Gregor, coords., Control de convencionalidad, interpretación conforme y diálogo jurisprudencial. Una visión desde América Latina y Europa. México, Porrúa-Universidad Nacional Autónoma de México, 2012, pp. 29-30. 
autoridad del ente jurisdiccional en sí misma no basta para sustentar el fallo. ${ }^{12}$

Pero además de su importancia desde el punto de vista argumentativo, el diálogo jurisprudencial, como fenómeno contemporáneo, al implicar la interacción entre tribunales de distintas jurisdicciones, busca un enriquecimiento mutuo en la construcción de soluciones equivalentes acordes a los principios universales del derecho democrático. ${ }^{13}$ De esta forma, la existencia de un diálogo jurisdiccional permite reforzar la legitimidad de las decisiones que se toman, facilitando su obediencia, lo que conecta con la filosofía de la democracia deliberativa, pues supone la existencia de varias autoridades que se encuentran comprometidas en un sistema de garantía de los derechos. ${ }^{14}$ Esto es así pues si bien, como se ha mencionado, tras conversar puede "alcanzarse un acuerdo entre los sujetos afectados, o un matizado disenso, pendiente de nuevos encuentros, o incluso un flagrante desacuerdo [...], en todo caso, se admite comúnmente entre las partes del sistema que todo diálogo produce al menos un recíproco enriquecimiento de las argumentaciones". ${ }^{15}$ No obstante, en ocasiones, la forma en que se produce el diálogo no es la mejor para fomentar ese enriquecimiento y, por ello, es conveniente encontrar elementos que permitan construir una tipología de comunicación judicial transnacional.

12 Bogdandy, Armin von, "Ius constitutionale commune latinoamericanum. Una aclaración conceptual”, en Fix-Fierro, Héctor, Bogdandy, Armin von y Morales Antoniazzi, Mariela, coords., Ius constitutionale commune en América Latina: Rasgos, potencialidades y desafíos, México, Universidad Nacional Autónoma de México-Max-Planck-Institut für ausländisches öffentliches Recht und Völkerrecht-Instituto Iberoamericano de Derecho Constitucional, 2014, p. 14.

13 Ayala Corao, Carlos, Del diálogo jurisprudencial al control de convencionalidad, México, Porrúa-Instituto Mexicano de Derecho Procesal Constitucional, 2013, p. XIII.

14 García Roca, Javier, op. cit., p. 196.

15 Idem. 
IV. Elementos para una tipología del diálogo Judicial TRANSNACIONAL

Si se toman en cuenta los usos y formas en que los jueces refieren sentencias extranjeras en sus resoluciones podemos encontrar algunos elementos para la conformación de una tipología de esta forma de comunicación judicial transnacional. En este sentido, y para comenzar con lo obvio, puede señalarse que una cita, presumiblemente, anticipa un contenido de interés para el lector. Esto significa que los jueces emplean las citas o referencias en su actuación por una razón específica: por su congruencia, por el rigor del análisis que en ella se emplea, por lo adecuado de sus conclusiones, por el estatus de un juez o de un tribunal o porque quieren persuadir a un auditorio específico de lo apropiado de los argumentos que desarrollarán. ${ }^{16}$ Sin embargo, existen diferentes formas en que los jueces utilizan las citas a sentencias extranjeras y de ello depende el peso que éstas tienen tanto dentro como fuera del tribunal que las utiliza.

\section{Atendiendo a las razones de su utilización}

En primer lugar, el recurso a jurisprudencia extranjera puede clasificarse tomando en cuenta los motivos por los que los jueces constitucionales recurren a las sentencias extranjeras. En este sentido, las citas pueden ser utilizadas por los jueces para demostrar un conocimiento básico de la materia que deben tratar. Al referir casos que son muy conocidos en una materia determinada, el juez envía el mensaje de que conoce los argumentos que, sobre el tema que examina, se han desarrollado en otras jurisdicciones. Esto hace que el juez, siempre que realice la referencia de manera adecuada, sea visto como una persona preparada.

16 McCormick, Peter y Praskach, Tammy, "Judicial Citation, the Supreme Court of Canada, and the Lower Courts: A Statistical Overview and the Influence of Manitoba", Manitoba Law fournal, vol. 24, núm. 2, 1996-1997, p. 338. 
Además, los jueces también pueden emplear las citas a precedentes extranjeros para situar el análisis que deben llevar a cabo en el contexto de principios y estándares establecidos por la comunidad internacional. Así, los tribunales refieren diversos casos resueltos en otras jurisdicciones para crear un horizonte de reconocimiento y, de esta manera, preparar y orientar su argumentación. ${ }^{17}$

Los tribunales usan también las sentencias extranjeras para integrar sus pautas interpretativas o agregar contenidos al derecho nacional. A través del uso de material jurisprudencial extra-sistémico, los jueces pueden implementar principios de interpretación que han sido empleados en otras jurisdicciones y, con ello, analizar los problemas que se les presentan desde una óptica diversa para entenderlos mejor y encontrar soluciones adecuadas al contexto local. Además, el uso de sentencias extranjeras también puede tener una vertiente funcionalista a partir de la cual los integrantes de los órganos jurisdiccionales encuentran diversas formas de resolver problemas comunes en las decisiones de otros jueces, obteniendo con ello el máximo provecho de la deliberación comparativa. De esta manera, el uso de precedentes extranjeros también sirve como una fuente de sugerencias para perfeccionar el derecho nacional. ${ }^{18}$ $\mathrm{Y}$ es que cuando el derecho de un determinado país debe ser completado colmando las lagunas que en éste existen o corrigiendo las

17 Lollini, Andrea, "La circolazione degli argomenti: método comparato e parametri interpretativi extra-sistemici nella giurisprudenza costituzionale sudafricana”, en Diritto Pubblico Comparato ed Europeo, núm. 1, 2007.

18 Rosenkrantz, Carlos F. ha señalado al respecto que: "Constitutional authorities sometimes use foreign law [...] as a source of legal innovation and knowledge. Foreign constitutional materials may liberate, helping us to jettison the conviction of false necessity that usually accompanies those who have been intellectually bred in the context of fixed institutions. An entire new range of ideas and possibilities may open up when we look abroad. New ways to deal with protracted and common problems may appear feasible." Rosenkrantz, Carlos F., "Against borrowings, and other nonauthoritative uses of foreign law", International fournal of Constitutional Law, vol. 1, núm. 2, 2003, p. 288. 
imprecisiones o divergencias que en él se encuentran, una forma de modernizarlo puede ser recurriendo al método comparativo en la interpretación del texto constitucional.

Las citas a jurisprudencia extranjera también pueden usarse para añadir peso y credibilidad a los argumentos que se analizan, haciéndolos más persuasivos y, por tanto, más aceptables para el auditorio al que están dirigidos. ${ }^{19}$ Las soluciones que los principales sistemas extranjeros dan a cuestiones delicadas que implican argumentos controvertidos (por ejemplo de índole ética o religiosa) son un importante punto de referencia para los tribunales nacionales que afrontan problemas en los que está interesada una opinión pública dividida. Calabresi y Zimdahl identifican las resoluciones a las que los tribunales dan este uso como "logical reinforcement cases". ${ }^{20}$ Bajo este esquema, los tribunales generalmente se basan en fuentes locales para llegar a su decisión, pero recurren al material extranjero para mostrar que su interpretación no es irracional o peculiar, con lo que añaden fuerza a sus resoluciones y, en cierto sentido, también las legitiman.

Los tribunales constitucionales en ocasiones utilizan también las sentencias extranjeras de manera probatoria. Esto puede suceder de manera negativa cuando, por ejemplo, un juez en un voto particular ofrece una interpretación divergente respecto a la de la

19 Ganesh Sitaraman, sobre este punto, ha dicho que: "The use of foreign law for persuasive reasoning argues that different nations may face similar situations and therefore one judge's analysis of a situation may be helpful to another judge elsewhere. These 'parallel rules' provide justification for considering foreign law. Persuasive reasoning involves a judge considering the argumentation or logic of a foreign decision and using that argument in his decision. The foreign case is not authoritative, but merely provides an example of an intelligent person reasoning through a legal problem perhaps similar to an academic article that seeks to analyze a problem and suggest an answer." Sitaraman, Ganesh, "The use and abuse of foreign law in constitutional interpretation", Harvard Fournal of Law \& Public Policy, vol. 32, núm. 2, 2009, p. 676.

20 Calabresi, Steven y Zimdahl Stephani, "The Supreme Court and foreign sources of law: Two hundred years of practice and the juvenile death penalty decision", William and Mary Law Review, vol. 47, 2005-2006, p. 899. 
mayoría para mostrar su desacuerdo con la resolución tomada; o bien en sentido positivo, cuando el tribunal o incluso un juez (en un voto concurrente) aporta argumentos tomados de una decisión extranjera para probar su hipótesis. Este esquema argumentativo está muy ligado al que acabamos de analizar y se basa, como señala Lollini, en la afirmación "incluso en el extranjero piensan así". Los jueces, por tanto, con el fin de reforzar una cierta interpretación afirman que ésta corresponde, se alinea o coincide con las de otro juez extranjero. Por lo tanto, el uso de parámetros extra-sistémicos tiene una función de reforzamiento de carácter retórico-probatorio. ${ }^{21}$ En el ámbito de la comparación probatoria se da un segundo esquema que se concreta en la afirmación: "visto que en el extranjero piensan así.... entonces" también nosotros atribuimos el mismo significado. Este esquema se diferencia del anterior porque a través de él se identifica un modelo hermenéutico extranjero al cual reconducir, posteriormente, la interpretación local. En este caso el fenómeno tiene consecuencias más problemáticas, pues existe un muy amplio número de interpretaciones que pueden tomarse en cuenta para desentrañar el "significado" de una norma constitucional o de los cuales derivar esquemas argumentativos. Por este motivo, en este segundo esquema, la discrecionalidad del juez juega un papel muy importante. Sin embargo, los peligros que puede implicar esta práctica son muchos pues el hecho de tomar el significado de los enunciados normativos internos de forma directa y automática de parámetros extrasistémicos significa indirectamente incorporar una "pseudo- fuente" al sistema nacional, ya que con esta práctica se produce una dinámica consecuencial: la interpretación de un determinado enunciado constitucional deriva automáticamente del parámetro extranjero mediante un procedimiento de subsunción de un caso particular a un precedente extra-sistémico. ${ }^{22}$

\footnotetext{
21 Lollini, Andrea, op. cit., p. 499.

22 Ibidem.
} 
Los tribunales también pueden citar precedentes extranjeros para establecer proposiciones fácticas que demuestren que una solución determinada ha funcionado en otros lugares. De esta forma, a través del análisis de los efectos que ha tenido una determinada sentencia en el extranjero, se puede saber si la finalidad de dictar una resolución determinada se cumple utilizando los argumentos que el tribunal piensa emplear. ${ }^{23} \mathrm{~A}$ partir de este uso también puede encontrarse una vertiente negativa o de resistencia, que se presenta cuando los jueces utilizan los precedentes extranjeros para darse cuenta de lo que no debe hacerse, pues las consecuencias de una determinada solución puede provocar problemas o simplemente porque la solución tomada en otros países no puede aplicarse al contexto local. ${ }^{24}$

Los jueces constitucionales en ocasiones usan también las sentencias extranjeras para ponderar determinadas soluciones con el fin de saber cuál es la más adecuada o si alguna de ellas puede aplicarse al contexto local. Bajo este esquema, los jueces toman en consideración propuestas de interpretación extranjeras diversas y ponderan las soluciones que sobre un problema determinado se han dado, delimitando la oscilación interpretativa para considerar o excluir de su razonamiento ciertos argumentos. A través de esta técnica, el tribunal busca la mejor interpretación entre varios esquemas ar-

23 Sobre este punto véase Fontana, David, "Refined comparativism in constitutional law", UCLA Law Review, núm. 49, 2001-2002, p. 555.

24 Según señala Rosenkrantz "Negative borrowing is a consequence of focusing on the 'failures of other constitutional regimes'. The reason for the rejection varies. Sometimes, it is done with a clear awareness of categorical differences between countries [...] At other times, differences in culture or national pride underlie the decision to refuse to resort to foreign models. Finally, a decision not to borrow may follow from a deep conviction about the implausibility of a given constitutional scheme. This motive is what Professor Scheppele calls 'aversive constitutionalism', where the construction of a constitutional text and a constitutional culture is based on the rejection of a particular conception of a constitution." Cfr. Rosenkrantz, Carlos F., op. cit., p. 289. 
gumentativos contrapuestos y determina si ésta es aplicable al caso que debe resolver o si alguno o ninguno de estos esquemas puede(n) aplicarse al contexto local. ${ }^{25}$ De esta forma, el uso de sentencias extranjeras puede servir también para enfatizar que los hechos particulares de un caso requieren una solución propia.

Los tribunales constitucionales, a través del uso de jurisprudencia extranjera, también intentan demostrar el desarrollo de un incipiente ius commune. Haciendo un recuento de las soluciones dadas en ciertos países, los jueces constitucionales en ocasiones tratan de determinar cuáles son los valores que rigen en las sociedades democráticas. Se dan casos también en los que a través del uso de material jurisprudencial extra-sistémico, los tribunales constitucionales intentan construir postulados de justicia global y reaccionar ante presiones externas. En estos supuestos, los tribunales constitucionales actúan como defensores transnacionales de ciertos valores.

Finalmente, las sentencias extranjeras pueden usarse también para entablar un diálogo entre jueces, aportando elementos argumentativos o de interpretación que son empleados por otras jurisdicciones, pero también tomando en cuenta la forma en que resuelven determinados problemas otros órganos jurisdiccionales de control.

\section{Atendiendo a la frecuencia}

La segunda clasificación que podemos hacer del recurso a sentencias extranjeras por parte de los jueces tiene que ver con la frecuencia en que los jueces recurren a esta práctica. En este grupo pueden darse

25 Choudhry señala al respecto que: "Through a process of interpretive selfreflection, courts may conclude that domestic and foreign assumptions are sufficiently similar to one another to warrant the use of comparative law. Conversely, courts may conclude that comparative jurisprudence has emerged froma fundamentally different constitutional order; this realization may sharpen an awareness of constitutional difference or distinctiveness." Choudhry, Sujit, Globalization in search of justification: Toward a theory of comparative constitutional interpretation", Indiana Law Review, vol. 74, 1999, p. 828. 
diversas hipótesis dependiendo del grado en que los tribunales recurren a jurisprudencia extranjera. Partiendo de uno de los extremos de la gradación, la primera hipótesis que se presenta es el no uso de precedentes extranjeros en la actividad interpretativa. Los tribunales, en este supuesto, ignoran por completo el derecho extranjero, pues no lo consideran útil al momento de llevar a cabo su labor interpretativa. En este caso se encuentran actualmente sólo pocos sistemas cerrados, pues el diálogo judicial transnacional tienen día a día con mayor fuerza hacia la universalización.

En un segundo grado podríamos encontrar el uso críptico o clandestino de sentencias extranjeras. Este uso, como señala Palermo, es un terreno difícil y resbaloso, pues en ausencia de citas expresas faltan pruebas inmediatas del razonamiento comparativo que realizan los jueces, y, sin embargo, la observación de los fenómenos de circulación de soluciones y técnicas jurisdiccionales con los lentes del método comparado ayudan a encontrar la operatividad que tiene el análisis comparado a fin de resolver controversias concretas. ${ }^{26} \mathrm{Y}$ es que en muchos casos en el trabajo de los jueces, a pesar de no existir una referencia explícita a precedentes extranjeros, se nota la influencia de resoluciones extra-sistémicas. El uso inexpreso del derecho extranjero, sin embargo, muchas veces es difícil de determinar y no en pocas ocasiones da al observador un excesivo margen de discrecionalidad para indicar (sobre todo en los ordenamientos que comparten la misma evolución liberal democrática) si principios, fórmulas, rationes decidendi o soluciones de diverso tipo son producto del trabajo de los tribunales locales o tienen su origen en una recepción pasiva. ${ }^{27}$ Es por esta razón que el uso clandestino

26 Palermo, Francesco, "La Corte costituzionale austriaca e la comparazione giuridica”, en Ferrari, Giuseppe Franco y Gambaro, Antonio (eds.), Corti nazionali e comparazione giuridica, Edizioni Scientifiche Italiane, Nápoles, 2006, p. 144.

27 Orrù, Romano, "La giustizia costituzionale in azione e il paradigma comparato: l'esperienza portoghese", en Ferrari, Giuseppe Franco y Gambaro, Antonio, op. cit., p. 5. 
ha sido criticado por personajes como Slaughter, quien ha señalado que el peor de todos los mundos sería que los jueces estuvieran muy influenciados, pero de manera secreta, por otras fuentes jurídicas. ${ }^{28}$

En un tercer grado se incluirían a las referencias explícitas a material extranjero que, a su vez, pueden dividirse en diversas categorías. La primera de ellas es definida por Alessandro Somma como el uso ornamental de la jurisprudencia extranjera. ${ }^{29}$ En este caso la cita culta, meramente ocasional, o en passant, sirve sólo para reforzar la convicción del juez de la bondad de la solución citada a manera de obiter dictum, pero no lo conduce a la solución que, de cualquier forma, habría encontrado por su cuenta aplicando el derecho nacional. ${ }^{30}$ En esta categoría

28 Slaughter, Anne Marie, "A brave new judicial world", en Ignatieff, Michael, (ed.), American Exceptionalism and Human Rights, Princeton University Press, Princeton, 2005, p. 297.

29 Somma, Alessandro, "Metodi e scopi della comparazione giuridica nelle decisioni delle corti", en Alpa, Guido (ed.), Il giudice e l'uso delle sentenze straniere. Modalitáà e tecniche della comparazione giuridica, Giuffrè, Milán, 2006.

3о Ibidem. Sobre este tipo de citas Sitaraman señala que: "Judges might cite foreign law or international law because they like a particular turn of phrase used. This type of citation is similar to cases in which the Court cites great works of literatura [Barnicy vs. Vopper Rehnquist, Dissenting, C.J., "Although the Court recognizes and even extols the virtues of this right to privacy, these are 'mere words'. W. Shakespeare, Troilus and Cressida, act V, sc. 3, owerridden by the Court's newfound right to publish unlawfully acquired information of public concern"] or pop music [Sprint Comm vs. APCG Services, Inc., (2008) Roberts, C.J. dissenting, 'The absence of any right to substantive recovery means that respondents cannot benefit from the judgment they seek an thus lack Article III standing'. 'When you got nothing, you got nothing to lose' Bob Dylan, Like a Rolling Stone, on Highway 61 Revisited (Columbia Records 1965)], and like citations of literature or music, this type of citation does not offend any significant values. It does not undermine expressive, democratic, or institutional competency values because the court is merely using words not their underlying reasoning, and the sources themselves are not authoritative. That Bob Dylan or William Shakespeare used the phrase is not the reason the court is adopting a certain rule. Nor is quoting language methodologically troubling. It is unnecessary to have a deep or broad knowledge of the foreign nation's laws merely to restate a phrase. Indeed, quoting language 
pueden incluirse, además de citas precisas, referencias generales (v. gr. a "jurisprudencia extranjera", a "los tribunales constitucionales de los países democráticos", o a "la jurisprudencia de los países liberal-democráticos”) que, muchas veces son poco útiles para un tribunal que pretenda utilizarlas y que en ocasiones crean problemas o confusiones a quienes toman en consideración estas referencias.

Los tribunales constitucionales también pueden emplear las sentencias extranjeras ad abundantiam. En estos casos, el uso tiene una finalidad que ayuda a la decisión. Los jueces a través de este esquema pueden añadir valor interpretativo a sus resoluciones, demostrar la construcción de una opinio communis o hacer patente la coincidencia de sus argumentos con los de la comunidad internacional.

El uso expreso de jurisprudencia extranjera también puede clasificarse de conformidad con la investigación comparativa que los jueces llevan a cabo. En este caso, se pone en consideración el grado de dominio con el que los jueces constitucionales se valen del método y de los instrumentos de la comparación. Rinella observa que en esta clasificación se toman en cuenta las hipótesis en las que la Corte revisa la legislación y la jurisprudencia extraña al ordenamiento jurídico de su país, para encontrar argumentos que le ayuden a sostener una determinada interpretación. La técnica empleada por la Corte varía en relación con el fin que se persigue, razón por la cual la investigación comparativa en ocasiones resulta puntual y sistemática, mientras que en otras es sumaria o fragmentaria. ${ }^{31}$

Los tribunales constitucionales a veces emplean también las sentencias extranjeras señalando de manera puntual el fin que desean perseguir a través del recurso al argumento comparativo, mientras

might actually prove to have benefits without drawbacks in that it signals to other nations that prominent American jurists read their opinions." Sitaraman, Ganesh, op. cit., pp. 664 y s.

31 Rinella, Angelo, "La Corte costituzionale del Sudafrica: il contributo del diritto comparato al consolidamento della democrazia", en Ferrari, Giuseppe Franco y Gambaro, Antonio, op. cit., p. 392. 
que en otras ocasiones el objetivo del uso del derecho comparado puede deducirse sólo en vía interpretativa y de acuerdo con el hilo conductor de la argumentación. ${ }^{32}$

La forma abierta en que se usan referentes jurisprudenciales extra-sistémicos también puede clasificarse de conformidad con los ordenamientos que un tribunal escoge al momento de llevar a cabo el análisis comparativo. Las razones que inducen a los jueces a referirse a algunos ordenamientos más que a otros son demasiado diversas y no siempre fundadas en motivos técnicos-jurídicos. En ocasiones pueden influir razones genealógicas (como la influencia de un ordenamiento en la proyección de otro o la importancia que tiene para países que han sido colonias de otras naciones el derecho que se aplicaba en ellos antes de su independencia) o incluso factores totalmente extraños a la dimensión jurídica como el prestigio internacional de un ordenamiento, el idioma en que están disponibles los materiales a los que se hará referencia o el currículo de los jueces constitucionales, sólo por mencionar algunos casos. ${ }^{33}$

Las referencias a sentencias extranjeras también pueden tener como finalidad demostrar algo. En estos casos las referencias tienen un carácter expresivo que intenta dejar claras ciertas situaciones. Por ejemplo, después de la caída de regímenes totalitarios, los tribunales constitucionales pueden encontrar en la jurisprudencia extranjera argumentos que hagan evidente que se ha roto con el pasado y que la voluntad de reconstruir un nuevo orden constitucional está presente en sus decisiones.

El uso expreso de material extranjero puede clasificarse también, de acuerdo con las sentencias que se tomen en cuenta, en diacrónico y sincrónico. Si éstas son actuales, la comparación será sincrónica, pero si sirven como referencias históricas, entonces el uso será diacrónico.

\footnotetext{
32 Ibidem.

33 Ibidem.
} 
También existen casos en los que el recurso a sentencias extranjeras es más problemático. El más obvio se presenta cuando la jurisprudencia de otros países se usa de manera errónea, debido a un equívoco o a una falta de entendimiento del precedente. Otro uso problemático se presenta cuando el tribunal otorga demasiada autoridad al precedente extranjero. En estos casos, el peso que los tribunales dan a los recursos extra-sistémicos es exagerado al grado que se siguen sin el menor análisis las decisiones de otros tribunales (más como un acto de fe que como resultado de un estudio comparativo). Además de estos casos, los jueces pueden recurrir a sentencias extranjeras por agregación, es decir, sin llevar a cabo un adecuado estudio comparativo, pero recurriendo al simple "consenso numérico" para sostener su posición.

Por otra parte, es necesario señalar que los tribunales también pueden hacer uso de sentencias extranjeras de manera indirecta, esto es, remitiendo a la jurisprudencia constitucional anterior que contiene referencias a sentencias extranjeras. Éste es, sin duda, uno de los supuestos en los que la sintonía de la jurisprudencia del tribunal local con la jurisprudencia extranjera se hace más evidente pues, a través de la remisión, los jueces constitucionales admiten (aunque no siempre expresamente) haber hecho suya en un momento anterior la jurisprudencia extranjera respecto de un determinado tema.

\section{Atendiendo a su justificación}

La tercera clasificación que podemos hacer del uso de sentencias extranjeras está ligada con su justificación. Desde este punto de vista, el recurso a jurisprudencia de otros países se justifica cuando la ley local y la extranjera están unidas por una relación genealógica; cuando los tribunales extranjeros operan en el contexto de procedimientos confiables y por lo tanto no es irracional dar cierto grado de autoridad a las decisiones adoptadas por esos órganos de control; cuando el consenso internacional sugiere una línea que es 
posible seguir; cuando un problema es común a muchos países y la comparación puede servir para encontrar soluciones homólogas siempre que existan las condiciones necesarias para llevarla a cabo; cuando la solución tiene un fin económico que excede los límites del sistema jurídico en que es aplicada, o cuando existe un diálogo marcado sobre cierto tema y se quiere participar en él. El uso de sentencias extranjeras puede clasificarse también de acuerdo con las causas que hacen que éste sea común ${ }^{34}$ o en virtud de los operadores jurídicos que propician esta práctica. ${ }^{35}$

Las combinaciones que pueden darse entre estos esquemas son muy variadas y, debido a que no existen reglas para elegir una forma en la que deben emplearse las sentencias extranjeras, los usos y el modo en que éstas se utilizan depende completamente del operador jurídico que recurre a ellas y, por tanto, en el caso de los tribunales, del valor que éstos órganos jurisdiccionales deseen darle al método comparativo.

\section{v. Conclusiones}

La tipología ofrecida sobre el uso de sentencias extranjeras que realizan los jueces tiene como fin abonar al desarrollo teórico de una práctica que puede ser una herramienta cargada de futuro en la labor interpretativa que llevan a cabo los tribunales constitucionales. Y puede serlo porque, si somos objetivos, debemos tener en cuenta que en nuestro actuar casi siempre buscamos fuentes que nos sirvan como inspiración y estímulo; fuentes que nos muestren el camino

34 Entre estas causas podemos señalar la existencia de un pluralismo marcado y simétrico (como en el caso de Canadá), la presencia de un plurilingüismo estructural (como sucede en Suiza) o el desarrollo de una fase de transición hacia la democracia o de descolonización acelerada (como en Sudáfrica).

35 Los jueces constitucionales pueden utilizar por iniciativa propia los materiales extranjeros, o pueden verse condicionados a analizarlos cuando los propone alguna de las partes en el procedimiento o un tercero (v. gr. los amici curiae). 
que debemos tomar para no partir de cero y que nos ayuden a resolver los problemas que enfrentamos. Si esto es así, entonces todo aquello que puede ofrecer al juez material serio para su trabajo debería ser considerado un instrumento útil en su actividad.

El material jurisprudencial extranjero, en este sentido, ofrece una gran cantidad de argumentos que pueden ser valiosos pues, como señalan Abrahamson y Fischer, las opiniones extranjeras pueden ofrecer puntos de vista importantes que de otra manera no serían tomados en cuenta. ${ }^{36}$ Esta idea, al parecer, ha sido acogida por varios órganos de control y hoy en día son muchos los tribunales que están participando en el diálogo judicial transnacional, contribuyendo así a la creación de un consenso internacional sobre ciertas materias que cada vez adquiere más peso. Con ello gana fuerza también la idea planteada por Zagrebelsky de optar por un derecho constitucional abierto en el que los jueces constitucionales interactúen para desarrollar, de una mejor manera, sus funciones. En este constitucionalismo abierto, sin embargo, el uso de sentencias extranjeras no significa la humillación de las constituciones nacionales, pues lo que se busca a través de esta práctica no es construir un caballo de Troya para afirmar una dictadura universalista de los derechos, sino emplear un importante instrumento que nos ayude a comprender mejor nuestras propias constituciones, dándoles un sentido a través de un marco de fondo en el cual pueden asumir un significado preciso desde la perspectiva de un determinado momento histórico. ${ }^{37}$ Entonces, para participar correctamente en la apertura del constitucionalismo debemos tener presente, como señala Häberle, que la individualidad culturalmente captada de un Estado no debe ser anulada por el "medio" o el "vehículo" de la comparación constitucional o de los derechos, pues entonces la pluralidad correría el riesgo de verse reducida a uniformidad. Las similitudes

36 Abrahamson, Shirley S. y Fischer, Michael J., "All the world's a courtroom: judging in the new millennium", Hofstra Law Review, vol. 26, 1997-1998, p. 287.

37 Zagrebelsky, Gustavo, La legge e la sua giustizia, Il Mulino, Bolonia, 2008, p. 404. 
textuales externas no deben ocultar las diferencias que proceden del contexto cultural de la Constitución que se interpreta y, por tanto, los contenidos (de derechos fundamentales) que se reciben por la vía de la comparación jurídica deben ser repensados en el contexto "propio" del Estado constitucional receptor. ${ }^{38}$

Para lograr este cometido, los jueces deben emplear la jurisprudencia extranjera como los trabajadores de la construcción emplean los andamios, es decir, como instrumentos en los que pueden apoyarse para llegar más lejos y para facilitar su trabajo, pero que nunca son parte de la obra final y que de ninguna manera condicionan la forma de lo que se construye. ${ }^{39}$ La recepción entonces no puede ser mecánica y, por tanto, es necesario conocer las diversas formas en que se está empleando el uso de sentencias extranjeras, pues si no se recurre a ellas de manera adecuada, esta práctica no podrá rendir los beneficios que es capaz de ofrecer.

\section{Fuentes}

Abrahamson, Shirley S. y Fischer, Michael J., "All the world's a courtroom: judging in the new millennium", Hofstra Law Review, vol. 26, 1997-1998.

Ackerman, Bruce, "The rise of world constitutionalism", Yale Law School Occasional Papers, núm. 4, 1996.

Ayala Corao, Carlos, Del diálogo jurisprudencial al control de convencionalidad, México, Porrúa-Instituto Mexicano de Derecho Procesal Constitucional, 2013.

BARAK, Aharon, "A judge on judging. The role of a Supreme Court in Democracy", en Harvard Law Review, núm. 116, 2002-2003.

38 Häberle, Peter, "Métodos y principios de la interpretación constitucional. Un catálogo de problemas", en Ferrer Mac-Gregor, Eduardo (coord.), Derecho procesal constitucional, $5^{\text {a }}$ ed., t. IV, Porrúa, México, 2006.

39 La metáfora es tomada de Rosenkrantz, Carlos F., op. cit., p. 295. 
Bogdandy, Armin von, "Ius constitutionale commune latinoamericanum. Una aclaración conceptual", en Fix-Fierro, Héctor, Bogdandy, Armin von y Morales Antoniazzi, Mariela, coords., Ius constitutionale commune en América Latina: Rasgos, potencialidades y desafios, México, Universidad Nacional Autónoma de México-Max-Planck-Institut für ausländisches öffentliches Recht und Völkerrecht-Instituto Iberoamericano de Derecho Constitucional, 2014.

Burgorgue-Larsen, Laurence, "La formación de un derecho constitucional europeo a través del diálogo judicial", en Alejandro Saiz Arnaiz y Eduardo Ferrer Mac-Gregor, coords., Control de convencionalidad, interpretación conforme y diálogo jurisprudencial. Una visión desde América Latina y Europa. México, Porrúa-Universidad Nacional Autónoma de México, 2012.

Calabresi, Steven y Zimdahl Stephani, "The Supreme Court and foreign sources of law: Two hundred years of practice and the juvenile death penalty decision", William and Mary Law Review, vol. 47, 2005-2006.

Canivet, Guy, "La pratica del diritto comparato nelle corti supreme", en Markesinis, Basil y Fedtke, Jörg, coords., Giudici e diritto straniero. La pratica del diritto comparato, Bolonia, Il Mulino, 2009.

Choudhry, Sujit, Globalization in search of justification: Toward a theory of comparative constitutional interpretation", Indiana Law Review, vol. 74, 1999.

Ferrajoli, Luigi, Principia Iuris. Teoria del diritto e della democrazia, t. 1, Laterza, Roma-Bari, 2007.

Fontana, David, "Refined comparativism in constitutional law", UCLA Law Review, núm. 49, 2001-2002.

García Roca, Javier, "El diálogo entre el Tribunal Europeo de Derechos Humanos y los tribunales constitucionales en la construcción de un orden público europeo", Teoría y Realidad Constitucional, núm. 30, 2012.

Häberle, Peter, "Métodos y principios de la interpretación constitucional. Un catálogo de problemas", en Ferrer Mac-Gregor, Eduardo (coord.), Derecho procesal constitucional, $5^{\text {a }}$ ed., t. IV, Porrúa, México, 2006. 
L'Heureux-Dubé, Claire, "The importance of dialogue: Globalization and the international impact of the Rehnquist Court", Tulsa Law Journal, vol. 34, 1998.

Lollini, Andrea, "La circolazione degli argomenti: método comparato e parametri interpretativi extra-sistemici nella giurisprudenza costituzionale sudafricana", en Diritto Pubblico Comparato ed Europeo, núm. 1, 2007.

MaCormick, Peter y Praskach, Tammy, "Judicial Citation, the Supreme Court of Canada, and the Lower Courts: A Statistical Overview and the Influence of Manitoba", Manitoba Law Fournal, vol. 24, núm. 2, 1996-1997.

OrRÙ, Romano, "La giustizia costituzionale in azione e il paradigma comparato: l'esperienza portoghese", en Ferrari, Giuseppe Franco y Gambaro, Antonio (eds.), Corti nazionali e comparazione giuridica, Edizioni Scientifiche Italiane, Nápoles, 2006.

Palermo, Francesco, "La Corte costituzionale austriaca e la comparazione giuridica", en Ferrari, Giuseppe Franco y Gambaro, Antonio (eds.), Corti nazionali e comparazione giuridica, Edizioni Scientifiche Italiane, Nápoles, 2006.

Rahdert, Mark C., "Comparative constitutional advocacy", en American University Law Review, vol. 56, núm. 3, 2006.

Rinella, Angelo, "La Corte costituzionale del Sudafrica: il contributo del diritto comparato al consolidamento della democrazia", en Ferrari, Giuseppe Franco y Gambaro, Antonio (eds.), Corti nazionali e comparazione giuridica, Edizioni Scientifiche Italiane, Nápoles, 2006.

Rosenfeld, Michael, "Constitutional migration and the bounds of comparative analysis", New York University Annual Survey of American Law, vol. 67, 2001.

Rosenkrantz, Carlos F., "Against borrowings, and other nonauthoritative uses of foreign law", International Fournal of Constitutional Law, vol. 1, núm. 2, 2003. 
Sitaraman, Ganesh, "The use and abuse of foreign law in constitutional interpretation", Harvard Fournal of Law \& Public Policy, vol. 32, núm. 2, 2009.

Slaughter, Anne-Marie, "A brave new judicial world", en Ignatieff, Michael, (ed.), American Exceptionalism and Human Rights, Princeton University Press, Princeton, 2005.

— , "Judicial globalization", Virginia Fournal of International Law, vol. 40, 1999-2000.

Somma, Alessandro, "Metodi e scopi della comparazione giuridica nelle decisioni delle corti", en Alpa, Guido (ed.), Il giudice e l'uso delle sentenze straniere. Modalitáà e tecniche della comparazione giuridica, Giuffrè, Milán, 2006.

Zagrebelsky, Gustavo, La legge e la sua giustizia, Il Mulino, Bolonia, 2008. 